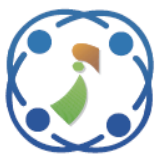

\title{
Iterative Receiver Compensation of HPA Nonlinearity in MIMO-GFDM System
}

\author{
Ari Endang Jayati ${ }^{1,2 *}$ \\ Wirawan Wirawan ${ }^{1}$ \\ Titiek Suryani $^{1}$ \\ Endroyono Endroyono ${ }^{1}$ \\ ${ }^{1}$ Department of Electrical Engineering, Faculty of Intelligent Electrical and Informatics Technology, \\ Institut Teknologi Sepuluh Nopember, Surabaya, Indonesia \\ ${ }^{2}$ Department of Electrical Engineering, Universitas Semarang, Semarang, Indonesia \\ * Corresponding author's Email: ndhank80@gmail.com
}

\begin{abstract}
Recently, various application scenarios have emerged in new communication networks that have a variety of new requirements such as: ultra-low latency, low out-of-band emission and long battery life. Generalized Frequency Division Multiplexing (GFDM) is one of the nonorthogonal multicarrier techniques that meets these requirements. We study the analysis of nonlinearity effects in a High Power Amplifier (HPA) and crosstalk on Multiple Input Multiple Output (MIMO)-GFDM systems and its compensation. Moreover, proposed is a recurrent receiver compensation method for the joint RF interference in the MIMO-GFDM system. The assumptions made at the receiver considered the characteristics of the HPA. This proposed technique also has the ability to compensate for the combined effects of nonlinear and crosstalk distortion. The simulation results show an improvement in the bit error rate for small iteration numbers, i. e. 1 - 4 iterations for 16-OQAM mapping. The receiver-based mitigation techniques were used as an option to maintain low-level transmitter complexity. The main advantage is the possible application at the base station without increasing the computational complexity of the mobile terminal as well as the terminal costs or battery life. This means the advanced nature of the technique ensured there was no need to make any changes on the cellular terminal side. Therefore, the performance of the iterative receiver technique proposed in the MIMO-GFDM system is better than other orthogonal multicarrier systems.
\end{abstract}

Keywords: High power amplifier, GFDM, MIMO, Crosstalk, Nonlinear distortion.

\section{Introduction}

With the emergence of various application scenarios in forthcoming communication networks, namely Fifth Generation (5G), various nonorthogonal multicarrier schemes are proposed to satisfy new requirements. GFDM is one of the nonorthogonal multicarrier techniques meets the requirements of future applications [1]. GFDM can be applied to Industrial applications 4.0 [2], Machine Type Communication (MTC) [3], Cognitive Radio [4,5], Satellite-Terrestrial [6] and Microwave Photonics [7]. The circular filter in GFDM retains the option to use Cyclic Prefix (CP) and Cyclic Suffix (CS) for the entire frame [8]. In addition, this filter avoids major delays and can minimize OOB emissions. For the low latency communications Industry, 4.0 short filter delay systems are an important advantage because latency reduction is a critical design criterion that GFDM can transmit [2].

A High Power Amplifier (HPA) is an important element of every communication system to communicate signals a significant distance [9]. Just like other multicarrier systems, GFDM also uses an HPA on the transmitter side. An HPA is used on the downlink system at the Base Station on a massive MIMO system for 5G technology [10]. HPAs are generally operated in linear regions close to the saturation region so that power efficiency is increased. When the HPA input power rises close to the saturation point, it increases power efficiency. But an HPA will produce nonlinear distortion if operated in this area [11]. Nonlinear distortion due to an HPA causes several effects including amplitude distortion, phase distortion, increase in out of band radiation, and spread of the signal constellation [12]. The nonlinear distortion effect also produces harmonic 
and intermodulation signals outside the frequency band which results in a spectral spread of the transmitted signal, in turn causing interference between adjacent subcarriers [11].

One of the main challenges in multicarrier systems such as the GFDM is the combined effect of nonlinear and crosstalk distortion due to the use of an HPA on an MIMO transmitter system [13] to convert low-power RF signals into high-power signals, which are required to drive the transmitter [14]. It is also applied on downlink systems at the Base Station on massive MIMO systems for 5G technology [10].

Crosstalk or coupling effect is the interference between signals on different paths which reduces the performance of the transmitted signal quality. However, it is possible to correct linear MIMO crosstalk using MIMO equalizers, though the nonlinear signals generated before HPA cannot be corrected by the receivers and are also considered to be very dangerous [15].

Several investigations have been conducted in recent years on PAPR, OOB radiation, and BER due to the exposure of GFDM systems to nonlinear distortion [16-18]. However, only a few have investigated techniques to deal with the effects of the distortion. Research has been conducted on how nonlinear HPA distortion affects GFDM [13, 19], but the combined effect with crosstalk on MIMO-GFDM systems was not examined.

To the best of our knowledge, there have been no studies investigating techniques for combining the effects of nonlinear and crosstalk distortion in MIMO-GFDM systems. Therefore, in this paper, we investigate the application of iterative receiver techniques to compensate joint nonlinear distortion and crosstalk in MIMO-GFDM systems.

The remaining sections of this report are organized as follows: Section 2 explains literature review. Section 3 presents the MIMO-GFDM system model with and without crosstalk and explains the proposed iterative receiver techniques and algorithms; Section 4 explains the results of the simulation and discussion, and Section 5 is a conclusion.

\section{Literature review}

Techniques to overcome nonlinear distortion due to HPA can be used on the transmitter or the receiver side. There are 4 types of techniques to overcome nonlinear distortion in multicarrier systems, namely reducing Input Back Off (IBO) [20], HPA linearization [21], PAPR reduction [16, 22, 23] and signal reconstruction on the receiver side [24].
Vaezi et. al. has investigated technique to overcome the combined effects of nonlinear and crosstalk distortion on MIMO-OFDM systems [25]. This study proposes the Digital Pre Distortion (DPD) technique. The HPA model used is polynomial memory (MP). This method works well for orthogonal modulation but has not been tried for nonorthogonal modulation.

Gregorio et. al. has proposed a digital selfinterference canceller on a full-duplex MIMO radio system but BER performance has not been investigated in this study [26]. Previous studies have been carried out [27] to overcome the combined effect of I / Q imbalance and crosstalk on MIMOOFDM systems in the transmitter. The method used in that case is MIMO-PD. Bassam et. al. proposed the CO-DPD method to compensate for non-linear crosstalk and distortion in MIMO systems on the transmitter but BER performance has not been investigated in this study [28].

Moreover, the techniques to overcome these combined effects have been researched [15] only in orthogonal multicarrier systems using OFDM while GFDM was performed in nonorthogonal ones [29]. The disadvantage of the predistorter technique is that it only compensates for nonlinear distortion. However, the interference in MIMO systems is not only due to this distortion but also crosstalk because of the different signal paths. Therefore, there is a need to study the techniques to compensate for the joint effects of nonlinear distortion and crosstalk in MIMO-GFDM systems.

The contributions of this paper are as follows:

1. First, the combined effects of HPA nonlinearity and crosstalk on MIMO-GFDM were simulated. The HPA was modeled by the famous Saleh model [30] which is an empirical model used in literature because of its ability to extract parameters using direct measurements.

2. Furthermore, an iterative receiver method was proposed to estimate and simultaneously reduce the combined effects, which was later applied at the base station for the uplink system. This technique assumes HPA is independent for each antenna and requires knowledge of its parameters.

3. Moreover, the BER performance of the iterative receiver method was demonstrated to compensate for the combined effects of nonlinear and crosstalk distortion on MIMO-GFDM systems.

\section{Proposed systems}

\subsection{System model}




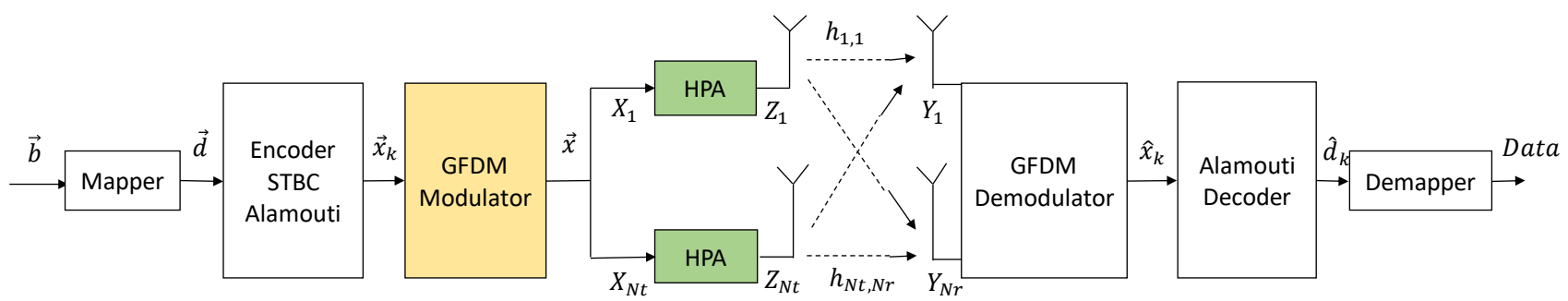

Figure. 1 MIMO-GFDM system with nonlinear HPA

\subsubsection{MIMO-GFDM wihout crosstalk}

The scheme of the MIMO-GFDM system without crosstalk is shown in Fig. 1. The information signal is passed through the mapper 16 Offset Quadrature Amplitude Modulation (OQAM), then the encoding process is done using STBC Alamouti. This encoder was chosen because it can reduce the signal-to-noise ratio (SNR). The output of the encoder is inserted in the GFDM modulator block.

Let $K$ and $M$ denote the number of subcarriers and sub-symbol samples for each GFDM block respectively. The results of the GFDM transmitter signal can be mathematically modeled as follows [31]:

$$
x(n)=\sum_{k=0}^{K-1} \sum_{m=0}^{M-1} g_{k, m}[n] d_{m, k}, n=0, \ldots, N-1
$$

with $N=K M$, and $g_{k, m}[n]$ is a shifted version of the Raised Cosine pulse shaping filter $g[n]$ in time (circular) and frequency as follows[32]

$$
g_{k, m}[n]=g\left[\left(n-\frac{m K}{2}\right) \bmod K M\right] e^{\frac{j 2 \pi k}{K}\left(n-\frac{L p-1}{2}\right)}
$$

whereas $d_{m, k}$ is the data transmitted by the $k$ subcarrier and sub-symbol to $m$ from each GFDM block. The GFDM output was given an HPA in each branch.

The HPA inputs for each branch are formulated as follows [15]:

$$
x_{k}=\rho_{k} e^{j \theta_{k}}
$$

where $\rho_{k}$ is the amplitude of $x_{k}$, and $\theta_{k}$ is the phase of the input $x_{k}$.

Therefore, the HPA output can be modeled as follows:

$$
\begin{gathered}
\boldsymbol{z}_{k}(n)=g\left[\boldsymbol{x}_{k}(n)\right]=A\left(\rho_{k}\right) e^{j\left(\theta_{k}+P\left(\rho_{k}\right)\right)} \\
=S\left(\rho_{k}\right) e^{j \theta_{k}}
\end{gathered}
$$

where $g$ [.] is the HPA transfer functions, $A($.$) and$ $P($.$) are \mathrm{AM} / \mathrm{AM}$ and AM/PM conversions on an HPA, and $S\left(\rho_{k}\right)$ is the amplitude of the HPA output. The Saleh Model with AM/AM characteristic functions were used and could be written as follows [30]:

$$
A[r(t)]=\frac{\alpha_{\alpha} r(t)}{1+\beta_{\alpha} r^{2}(t)}
$$

where $r(t)$ is the amplitude of the HPA input while the functional characteristics of AM/PM are as follows:

$$
\Phi[r(t)]=\frac{\alpha_{\varphi} r^{2}(t)}{1+\beta_{\varphi} r^{2}(t)}
$$

with $\alpha_{\alpha}, \beta_{\alpha}, \alpha_{\varphi}, \beta_{\varphi}$ being the parameters of the Saleh model.

The operating points on the HPA are usually identified using 'back-off' for validating theoretical results, which are defined as:

$$
I B O=10 \log _{10}\left(\frac{A_{0}^{2}}{P_{0}}\right)
$$

where $A_{0}$ is the saturation amplitude of the HPA input and $P_{0}$ is the average power input. From Bussgang's theorem and by extending complex Gaussian processes the output from HPA can also be represented as [15]:

$$
\boldsymbol{z}_{k}(n)=K_{0} \boldsymbol{x}_{k}(n)+\boldsymbol{d}_{k}(n)
$$

where $K_{0}$ is the attenuation coefficient, according to Table 1 in [15] the value of $K o$ is $0.7556+0.1643 \mathrm{i}$. $d_{k}(n)$ is a nonlinear distortion that does not correlate with the $x_{k}(n)$ signal input. The $K_{0}$ value is given by [15] with:

$$
K_{0}=\frac{1}{2} E\left[S^{\prime}\left(\rho_{k}\right)+\frac{S\left(\rho_{k}\right)}{\rho_{k}}\right]
$$




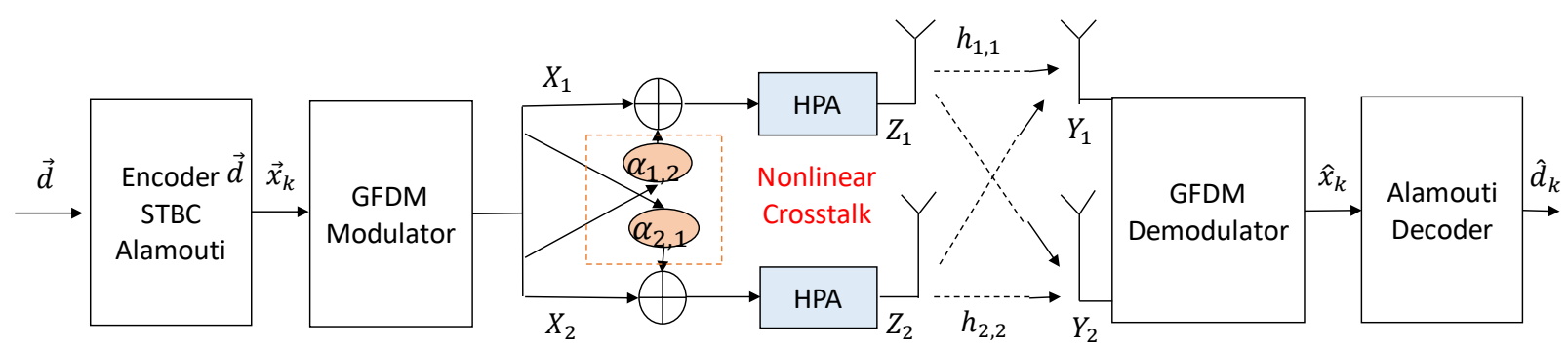

Figure. 2 MIMO-GFDM system with nonlinear crosstalk

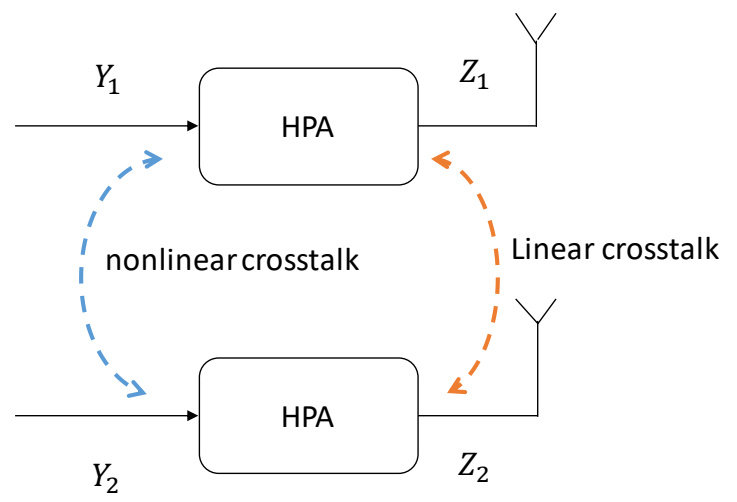

Figure. 3 Linear and nonlinear crosstalk

where $S^{\prime}\left(\rho_{k}\right)$ is denoted as a derivative of $S\left(\rho_{k}\right)$. The variance of the nonlinear distortion $d_{k}(n)$ are as follows [15]:

$$
\begin{gathered}
\sigma_{d}^{2}=E\left[\left|\boldsymbol{d}_{k}(n)\right|^{2}\right] \\
=E\left[\left|\boldsymbol{z}_{k}(n)\right|^{2}\right]-\left|K_{0}\right|^{2} E\left[\left|\boldsymbol{x}_{k}(n)\right|^{2}\right] \\
=E\left[\left|S\left(\rho_{k}\right)\right|^{2}\right]-\left|K_{0}\right|^{2} E\left[\rho_{k}^{2}\right]
\end{gathered}
$$

The signal received by each antenna $\boldsymbol{y}_{m}^{N L}(n)$ is replaced by the superposition of an independent fading signal associated with the $N_{t}$ antenna that shares the same frequency space. The received signal at time instant $n$ is affected by Gaussian noise on the array element as follows [15]:

$$
\begin{gathered}
\boldsymbol{y}_{m}^{N L}(n)=\sum_{k=1}^{N_{t}} \boldsymbol{H}_{m, k}(n)\left(K_{0} x_{k}(n)+\boldsymbol{d}_{k}(n)\right)+ \\
\boldsymbol{n}_{m}(n)
\end{gathered}
$$

where $\boldsymbol{n}_{m}(n)$ is Additive White Gaussian Noise (AWGN) and $\boldsymbol{H}_{m, k}(n)$ is a circular time-domain matrix $N_{c} \times N_{c}$ at time n, which is formed from the channel response vector $h_{m, k}(n)$ for the link between the sending antenna $k$ and the receiving antenna $m$.

The signal received in vector form for each antenna in the $k$-th subcarrier can be written as follows [15]:

$$
\begin{array}{rl}
\boldsymbol{Y}^{N L}(n, k)=K_{0} & \mathcal{H}(n, k) \boldsymbol{X}(n, k)+\mathcal{H}(n, k) \boldsymbol{D}(n, k) \\
& +\mathcal{N}(n, k)
\end{array}
$$

where $\mathcal{H}(n, k)$ is the channel transfer matrix in the frequency domain, $\boldsymbol{X}(n, k)=$ $\left[X_{1}(n, k) \ldots \ldots, X_{N t}(n, k)\right]^{T}$ is a vector containing the signal transmitted by each antenna and $\mathcal{N}(n, k)$ are Gaussian noise obtained by the FFT process of thermal Gaussian noise with zero mean and variance $\sigma^{2}$.

\subsubsection{MIMO-GFDM wihout crosstalk}

The MIMO-GFDM system scheme with crosstalk is shown in Fig. 2. The signal received after exposure to crosstalk can also be written in the form of a matrix as follows:

$$
\boldsymbol{y}=\boldsymbol{H} \boldsymbol{A}_{T} \boldsymbol{Z}+\boldsymbol{n}
$$

where $\boldsymbol{A}_{T}$ are the $N_{t} x N_{t}$ crosstalk matrix on the transmitter.

$$
\boldsymbol{A}_{T}(k, m)=\left\{\begin{array}{rc}
1, & k-m=0 \\
\alpha_{T}, & |k-m|=1 \\
0, & \text { others }
\end{array}\right.
$$

$\alpha_{T}$ represents crosstalks between adjacent signal paths on the transmitter. $\boldsymbol{Z}$ is a symbol transmitted with an average power of $P_{o}, n$ refers to the vector noise $n_{r} x 1$ with elements that are independent and identically distributed (iid). Complex circular Gaussian distribution $\mathcal{C N}\left(0, N_{0}\right)$ does not correlate with the transmitted symbol, and $H=\left[h_{m, k}\right]_{m, k=1}^{N_{R}, N_{T}}$ expresses the random channel matrix $N_{r} x N_{t}$ with $h_{m, k}$ representing the channel coefficient between the $m$-th transmitter antenna and the $k$-th receiver antenna[33].

Fig. 3 explains the types of crosstalk on MIMO systems. Linear MIMO crosstalk, which is relatively benign because it is being repaired by an MIMO equalizer, and nonlinear crosstalk that is not corrected by the MIMO receiver and is therefore far more dangerous [15].

Moreover, the HPA output with crosstalk effects can be written as follows [15]: 


$$
\begin{aligned}
& z_{k}(n)=g\left[x_{k}(n)+\sum_{l=1, l \neq k}^{N t} \alpha_{l k}(n) \otimes x_{l}(n)\right] \\
= & K_{0} x_{k}(n)+K_{0} \sum_{l=1, l \neq k}^{N t} \alpha_{l k}(n) \otimes x_{l}(n)+d_{k}(n)
\end{aligned}
$$

where $\otimes$ denotes the Kronecker product, $g$ [.] is the HPA response per branch, $x_{l}(n)$ is the output of GFDM from $l$ transmitter paths, and $\alpha_{l k}(n)$ is a filter representing the crosstalk and coupling modeling between $l$ to $k$ paths [27]. Finally, the signal received, if given a nonlinear crosstalk, is represented by:

$$
\begin{aligned}
y_{m}^{N L+\operatorname{cross}}(n)= & \sum_{k=1}^{N t} H_{m, k}(n) x\left(K_{0} x_{k}(n)\right. \\
& +K_{0} \sum_{l=1, l \neq k}^{N t} \alpha_{l k}(n) \otimes x_{l}(n) \\
& \left.+d_{k}(n)\right)+n_{m}(n)
\end{aligned}
$$

where $n_{m}(n)$ is Additive White Gaussian Noise (AWGN) and $H_{m, k}(n)$ is a circular time-domain matrix $N_{c} \times N_{c}$ at time $n$, which is formed from the channel response vector $h_{m, k}(n)$ for the link between the sending antenna $k$ and the receiving antenna $m$.

\subsection{Proposed methods}

Besides a predistorter technique to overcome nonlinear distortion in MIMO GFDM, also proposed is a mitigation technique at the receiver side is also proposed. Nonlinear Distortion Cancelation (NDC technique) is a nonlinear distortion mitigation method in MIMO systems with an iterative receiver technique that requires knowledge of HPA parameters[15]. This technique can overcome the combination of nonlinear distortion and HPA crosstalk problems in MIMO and it does not complicate the cellular terminal.

The analysis presented in this section is based on the assumption that the distortion caused by HPA can be modeled as a Gaussian additive noise, whose variance depends on the input signal and non-linear HPA characteristics. The Zero Forcing weight function is modeled as:

$$
\boldsymbol{W}_{(Z F)}=\left[\boldsymbol{H}^{H}(n, k) \boldsymbol{H}(n, k)\right]^{-1} \boldsymbol{H}^{H}(n, k)
$$

After equalization, the estimated signal can be obtained as:

$$
\widehat{\boldsymbol{X}}(n, k)=\boldsymbol{W}_{(Z F)} \boldsymbol{Y}(n, k)
$$

Table 1. Notation List of Proposed Methods

\begin{tabular}{|c|c|}
\hline Symbol & Description \\
\hline $\boldsymbol{W}_{(Z F)}$ & ZF weight function \\
\hline$\gamma$ & Instantaneous SNR \\
\hline$\sigma_{x}^{2}$ & Variance of signal \\
\hline$\sigma_{d}^{2}$ & $\begin{array}{c}\text { Variance of nonlinear } \\
\text { distortion }\end{array}$ \\
\hline$\sigma_{n}^{2}$ & Noise variance \\
\hline$e r f c$ & $\begin{array}{c}\text { Complementary error } \\
\text { function }\end{array}$ \\
\hline $\boldsymbol{H}^{H}$ & Conjugate transpose \\
\hline
\end{tabular}

Applying eq. (17) to eq. (12), the transmitted signal is obtained :

$$
\begin{aligned}
& \widehat{\boldsymbol{X}}(n, k) \\
& =\left[\boldsymbol{H}^{H}(n, k) \boldsymbol{H}(n, k)\right]^{-1} \boldsymbol{H}^{H}(n, k)\left[K_{0} \boldsymbol{H}(n, k) \boldsymbol{X}(n, k)\right. \\
& +\boldsymbol{H}(n, k) \boldsymbol{D}(n, k)+\mathcal{N}(n, k)]
\end{aligned}
$$

SNR for nonlinear cases is written as follows:

$$
\gamma=\frac{\gamma_{c} g(\gamma)}{g(\gamma) \sigma_{d}^{2}+\sigma_{n}^{2}}
$$

We considered that $\gamma_{c}=\left[K_{0}\right]^{2} \sigma_{x}^{2}, g(\gamma)=$ $\frac{\gamma \sigma_{n}^{2}}{\gamma_{c}-\gamma \sigma_{d}^{2}}$ and $g^{\prime}(\gamma)=\frac{\gamma_{c} \sigma_{n}^{2}}{\left(\gamma_{c}-\gamma \sigma_{d}^{2}\right)^{2}}$.

BER from MIMO-GFDM nonlinear OQAM becomes:

$$
\begin{aligned}
p_{N L}(e)=2\left(\frac{k-1}{k}\right) & \operatorname{erfc}(\sqrt{\gamma}) \\
& +-\left(\frac{k-1}{k}\right) \operatorname{erfc}^{2}(\sqrt{\gamma})
\end{aligned}
$$

\subsubsection{Iterative receiver without crosstalk}

Algorithm 1 is an iterative receiver algorithm without the crosstalk. This technique requires knowledge of HPA parameters. The proposed method is applied at the receiver side, separately for each antenna using its HPA parameters. Nonlinear distortion $d$ cannot be handled by conventional receivers. In practice, the recipient does not know d, but it must be known in order to get an estimate of the characteristics of the HPA model. Provided the receiver knows the nonlinear function of the transmitter $g(\cdot)$ of the HPA, it can be estimated from $y$. This is done repeatedly and can be described as follows: 


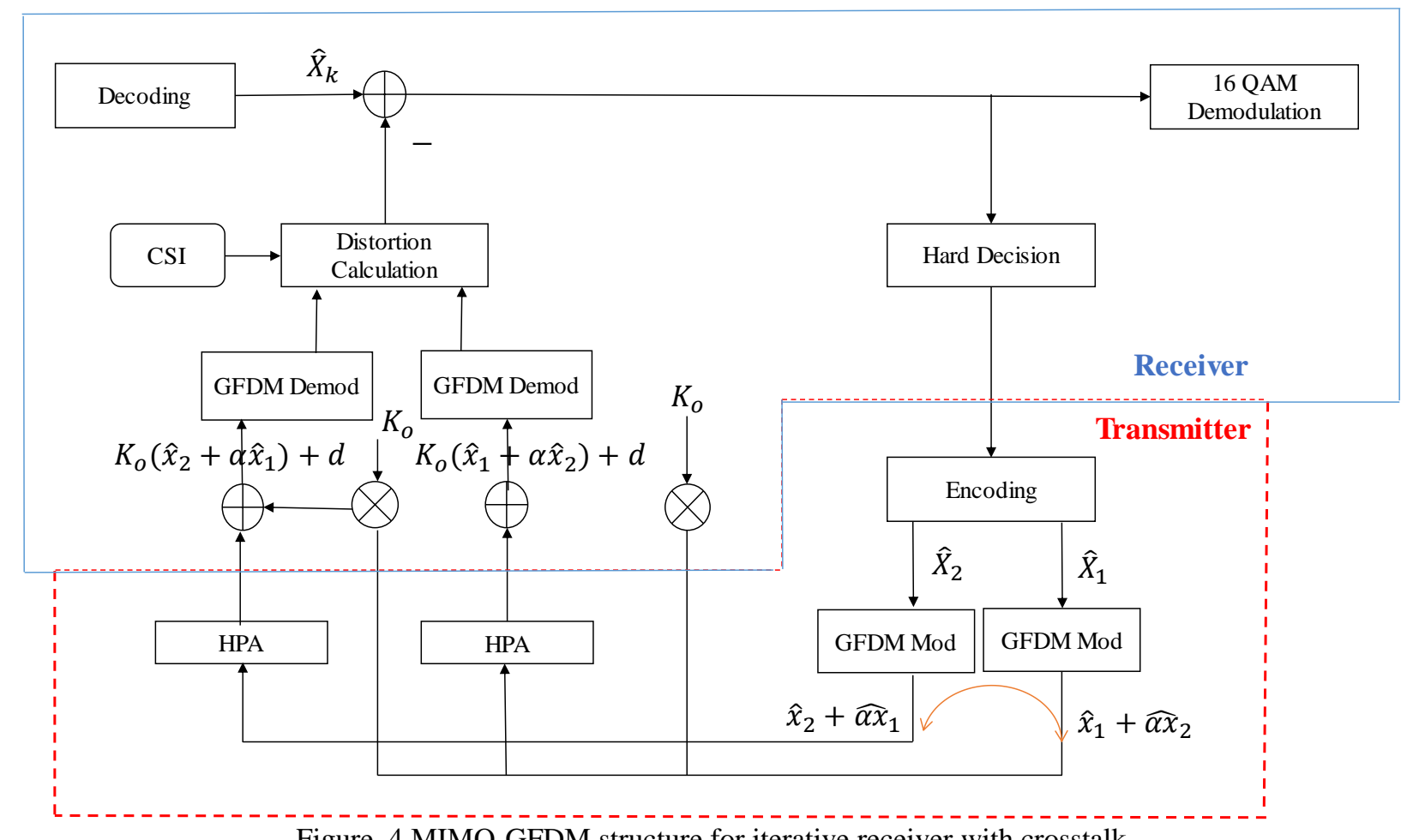

Figure. 4 MIMO-GFDM structure for iterative receiver with crosstalk

1. From the received signal, calculate the data symbol with a hard decision:

$$
\widehat{\boldsymbol{a}}^{(q)}=\left\langle\Gamma^{-1}\left(\boldsymbol{y}-\boldsymbol{d}^{(q-1)}\right)\right\rangle
$$

where $\langle$.$\rangle indicates a hard decision, \Gamma(.)^{-1}$ is a GFDM demodulation operation and $q$ is an iteration number. The first iteration of each element $d$ is set to zero and the data symbol is calculated directly from the received signal, i.e. $\widehat{\boldsymbol{a}}^{(1)}=\langle y\rangle$

2. Calculate the distortion as follows

$$
\begin{gathered}
\widehat{\boldsymbol{d}}_{k}^{(q)}(n)=\hat{g}\left[\widehat{\boldsymbol{x}}_{k}^{(q)}(n)\right]-K_{0} \widehat{\boldsymbol{x}}_{k}^{(q)}(n) \\
\text { with } \widehat{\boldsymbol{x}}_{k}{ }^{(q)}=\Gamma\left(\widehat{\boldsymbol{a}}^{(q)}\right)
\end{gathered}
$$

where $\Gamma($.$) is a GFDM modulation operation.$

3. Move to Step 1 and calculate $\widehat{\boldsymbol{a}}^{(q+1)}$ using both the received signal $y$ and the nonlinear distortion $\boldsymbol{d}^{(q)}$.

Using the results of Eq. (23), the transmitted constellation is estimated again by canceling the new distortion/decoding, and the process can be done iteratively. The process is terminated if $B E R(i+1)=$ BER (i).

\subsubsection{Iterative receiver with crosstalk}

The proposed MIMO-GFDM receiver structure with Iterative Receiver can be seen in Fig. 4. The iterative receiver method was applied at the receiver section of the system, and the workings of this recipient's proposal are as follows:

1. From the received signal, calculate the data symbol with a hard decision:

$$
\widehat{\boldsymbol{a}}^{(q)}=\left\langle\Gamma^{-1}\left(\boldsymbol{y}-\boldsymbol{d}^{(q-1)}\right)\right\rangle
$$

where $\langle$.$\rangle denotes a hard decision, \Gamma(.)^{-1}$ is a GFDM demodulation operation and $q$ is an iteration number. The first iteration of each element $d$ was set to zero and the data symbol calculated directly from the received signal, i.e. $\widehat{\boldsymbol{a}}^{(1)}=\|\boldsymbol{y}\|$

2. Calculate the distortion as follows:

$$
\begin{array}{r}
\widehat{\boldsymbol{d}}_{k}^{(q)}(n)=\hat{g}\left[\widehat{\boldsymbol{x}}_{k}^{(q)}(n)+\sum_{l=1, l \neq k}^{N t} \alpha_{l k}(n) \otimes\right. \\
\left.\hat{x}_{l}^{(q)}(n)\right]-K_{0} \widehat{\boldsymbol{x}}_{k}^{(q)}(n)+\sum_{l=1, l \neq k}^{N t} \alpha_{l k}(n) \otimes \hat{x}_{l}^{(q)}(n)
\end{array}
$$

$$
\text { where }: \widehat{\boldsymbol{x}}_{k}^{(q)}=\Gamma\left(\widehat{\boldsymbol{a}}^{(q)}\right)
$$




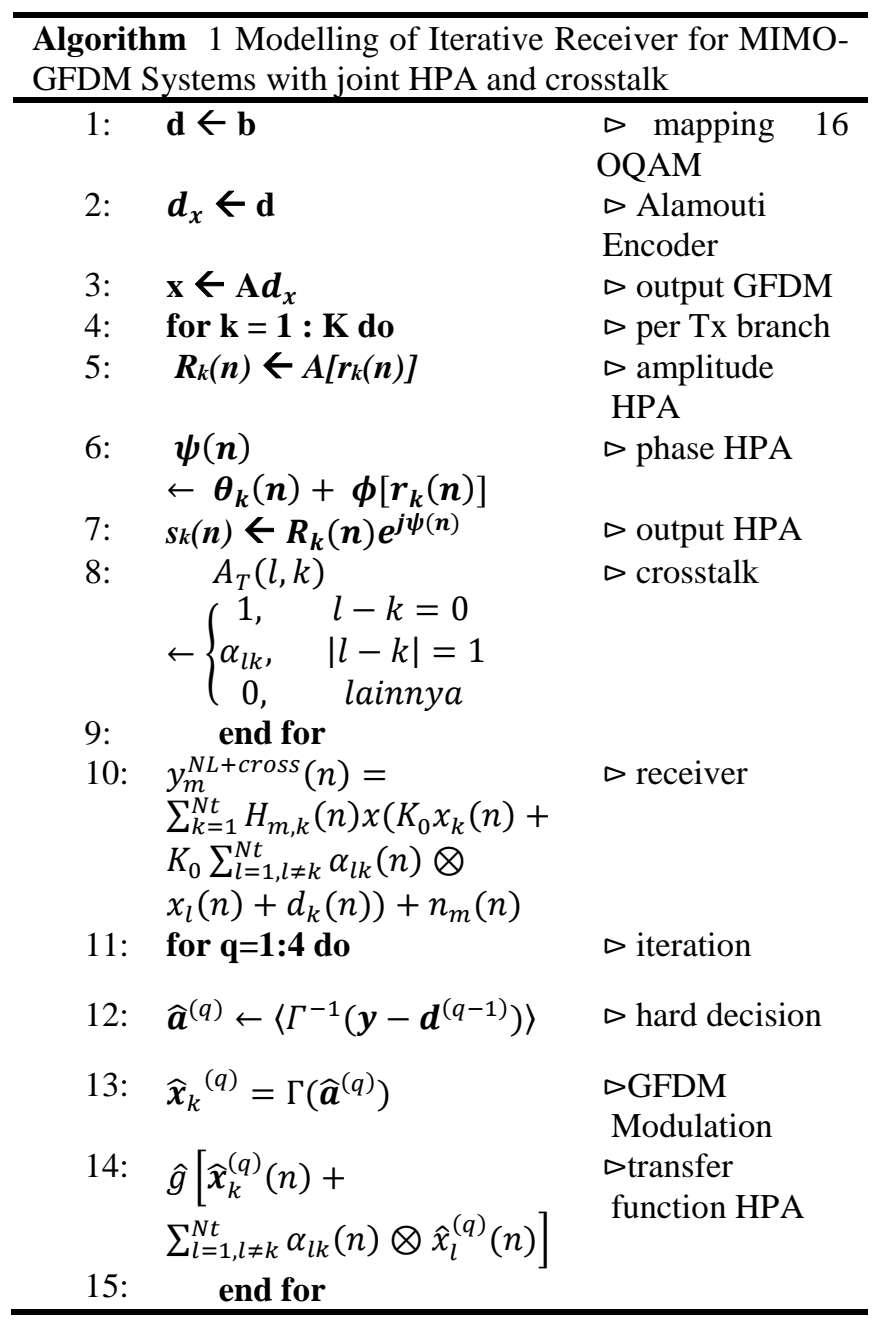

Table 2. GFDM Simulation Parameters with Iterative Receiver

\begin{tabular}{|l|c|l|}
\hline Parameter & Notation & GFDM \\
\hline Sampling Frequency & $f_{s}$ & 4096 \\
\hline Subcarrier & $\mathrm{K}$ & 8 \\
\hline Symbols per block & $\mathrm{M}$ & 32 \\
\hline Samples per symbol & $\mathrm{N}$ & 10 \\
\hline Pulse Shaping & $\mathrm{g}$ & RRC \\
\hline Roll-of-factor & $\alpha$ & 0,25 \\
\hline Alpha_a & $\alpha_{a}$ & 2.1587 \\
\hline Alpha_phi & $\alpha_{\varphi}$ & 4.0033 \\
\hline Beta_a & $\beta_{a}$ & 1.1517 \\
\hline Beta_phi & $\beta_{\varphi}$ & 9.104 \\
\hline Mapping & & $16 \mathrm{QAM}$ \\
\hline IBO & & $7 \mathrm{~dB}$ \\
\hline Crosstalk & & $5 \mathrm{~dB}$ \\
\hline
\end{tabular}

where $\Gamma($.$) is a GFDM modulation operation$ and $\hat{g}[$.$] is a nonlinear function.$

3. Move to Step 1 and calculate $\widehat{\boldsymbol{a}}^{(q+1)}$ using both the received signal $y$ and the nonlinear distortion $\boldsymbol{d}^{(q)}$. The iteration process ends after BER $(i+1)=$ BER (i) or the error rate is appropriate from the application point of view.

The algorithm method of iterative receivers proposed is written in Algorithm 1, consisting of three main steps as shown in Eqs. (25)-(27). The number of iterations is varying, depending on the constellation number of baseband modulation.

\section{Result and discussion}

Application of the iterative receiver method to mitigate the combined effects of nonlinear and crosstalk distortion in MIMO-GFDM systems has been investigated. The analysis covered includes system performance (namely BER), a constellation diagram, and signal spectrum before and after a combination of distortions. The research scenario follows the parameters in Table 2.

With a scheme like in Fig. 2, the crosstalk values vary from $5 \mathrm{~dB}, 10 \mathrm{~dB}$, to $20 \mathrm{~dB}$. This research investigates the combined effects of nonlinear and crosstalk distortion on MIMO-GFDM systems including spectrum, constellation, and BER. Next, it proposes an iterative receiver method which aims to estimate and reduce simultaneously the combined effects of HPA nonlinear and crosstalk distortion on the receiver side of the MIMO-GFDM system. The proposed method is applied at the Base Station (MS) for the uplink system. This technique requires knowledge of HPA parameters and HPA is assumed to be independent for each antenna.

The system with crosstalk is written as Algorithm 1. This algorithm consists of the main steps as written by Eqs. (22), (23), (25), and (26).

Crosstalk or coupling effect is the interference between signals with different paths and results in a decrease in the quality of the transmitted signals. This type of crosstalk can be linear and nonlinear. Linear MIMO crosstalk can be corrected by MIMO equalizers, while nonlinear crosstalk generated before HPA cannot be corrected by MIMO receivers and is very dangerous [15]. The problem becomes more complicated when the problem of nonlinear distortion due to HPA in MIMO GFDM is combined with use of high M-ary modulation.

This proposed method requires knowledge of HPA parameters, such as complex attenuation coefficients. Thus, distortion can be estimated with the knowledge of the HPA model and then calculated by subtracting the estimated signal from the original signal received. This procedure can be repeated iteratively.

The advantage of the chosen technique is that it can be implemented at the base station. Therefore, it does not increase the computational complexity of cellular terminals, so the results do not increase terminal costs 


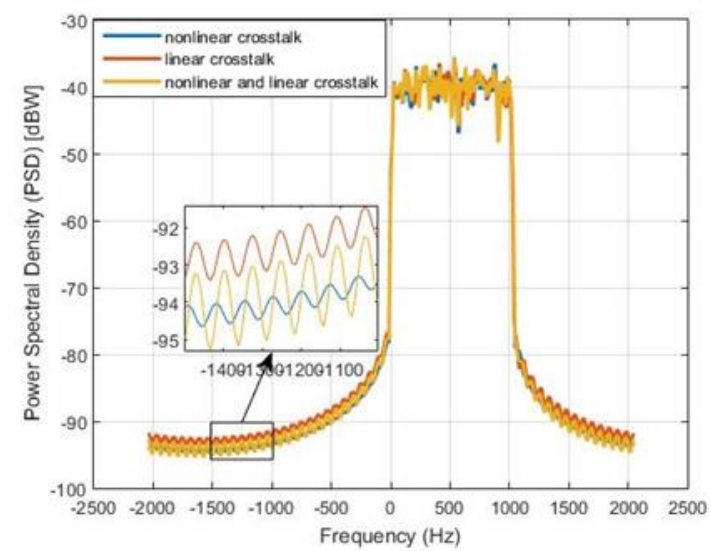

Figure. 5 Spectrum of MIMO-GFDM with and w/o crosstalk

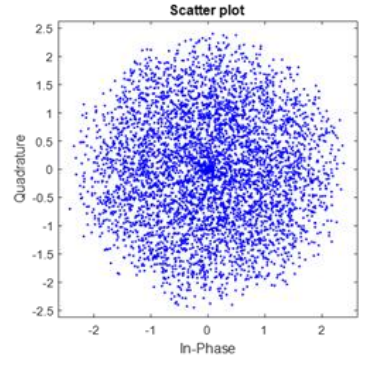

(a)

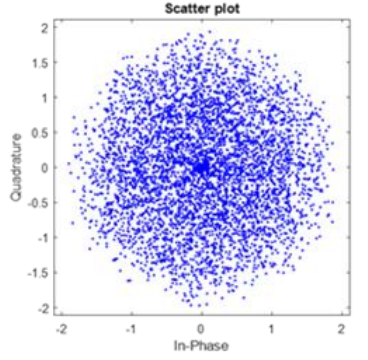

(b)

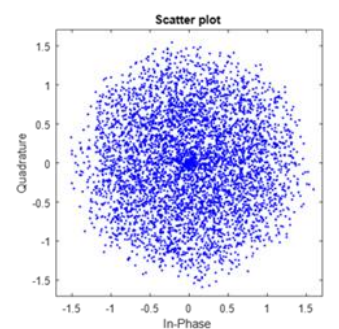

(c)

Figure. 6 Constellation for Crosstalk: (a) $5 \mathrm{~dB}$, (b) 10 $\mathrm{dB}$, and (c) $20 \mathrm{~dB}$

or battery time. On the other hand, the biggest loss problem is important to know the required knowledge of the exact parameters of the AM / AM and AM / PM characteristics of the HPA. This severely limits the practical work of the proposed scheme.

In Fig. 5 for MIMO-GFDM systems with nonlinear crosstalk, it can be seen that there are ripples in the inband section but OOB is low. Whereas for systems with linear crosstalk there are not many ripples in the inband section and OOB is also low.

Next the constellation diagram is observed after the system with an HPA and nonlinear crosstalk is added. This can be seen in Fig. 6 that the constellation diagram is seen spreading and deformation. Crosstalk variation causes different constellation diagram sizes; for a crosstalk value of $-20 \mathrm{~dB}$, the constellation diagram size gets smaller.

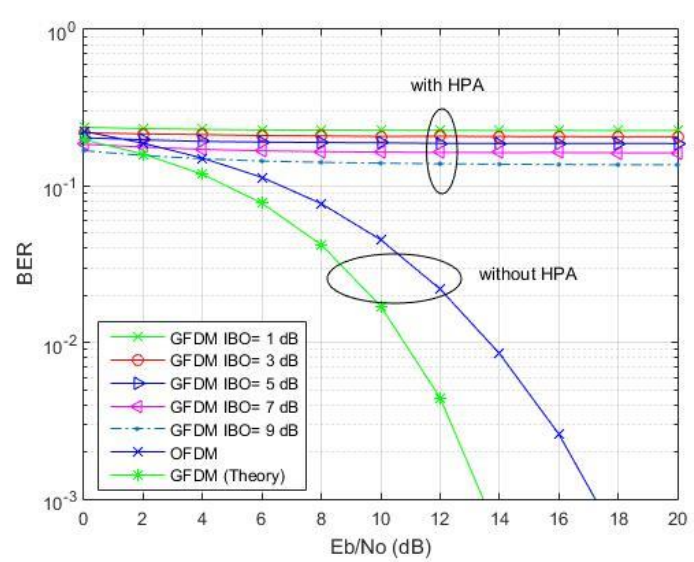

Figure. 7 Simulation and theory results for BER vs SNR on MIMO-GFDM systems (NL IBO variations)

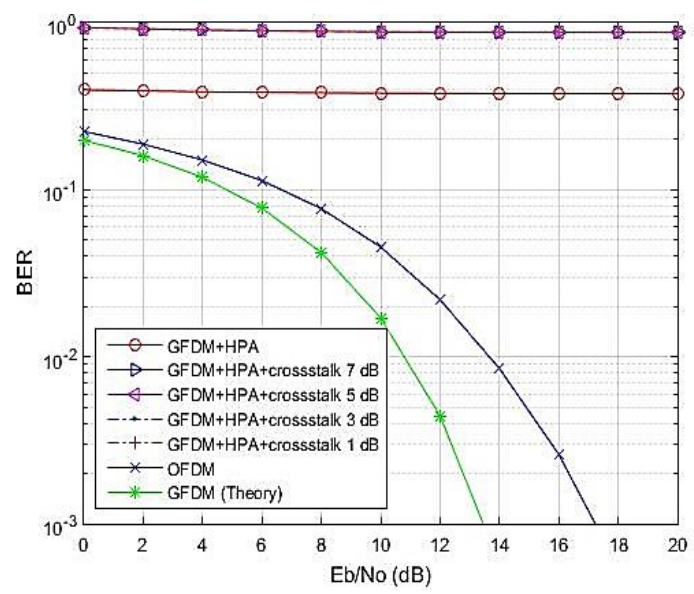

Figure. 8 Simulation results and theory for BER vs SNR on MIMO-GFDM systems (NL crosstalk variation)

In the third scenario, the effects of nonlinear distortion on the performance of the MIMO-GFDM system were investigated and the result is presented in Fig. 7. The effects of nonlinear distortion were found to become worse as the Input Back Off (IBO) value became smaller because the curve approaches the saturation point, decreasing system performance.

Furthermore, there is the BER comparison analysis between MIMO-GFDM systems without and with crosstalk. Fig. 8 shows the BER performance for the MIMO-GFDM system with crosstalk, the results look worse. This is because nonlinear crosstalk makes inband rippling. Therefore, systems with the addition of crosstalk have worse BER performance compared to those with only an HPA.

Fig. 9 illustrates BER versus $\mathrm{Eb} / \mathrm{No}$ for the performance of iterative receiver techniques in MIMO-GFDM systems. Two scenarios were observed: the first was the iterative receiver technique without crosstalk, the second being with crosstalk. As illustrated in Fig. 9 (a) and (b), the systems with the crosstalk have worse performance than those without 


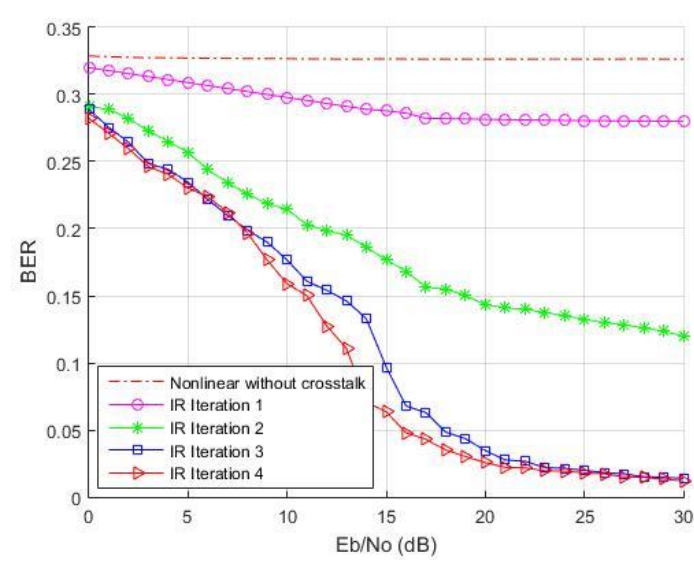

(a)

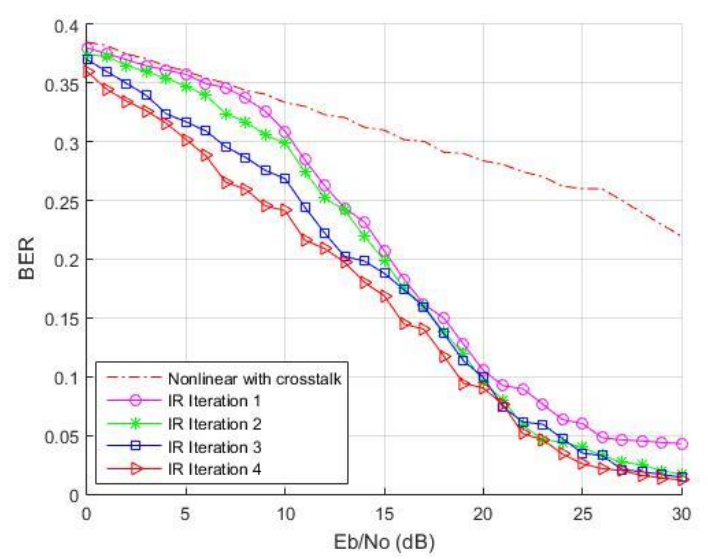

(b)

Figure. 9 Simulation results of the iterative receiver method on the MIMO-GFDM systems with and without crosstalk: (a) GFDM with HPA without

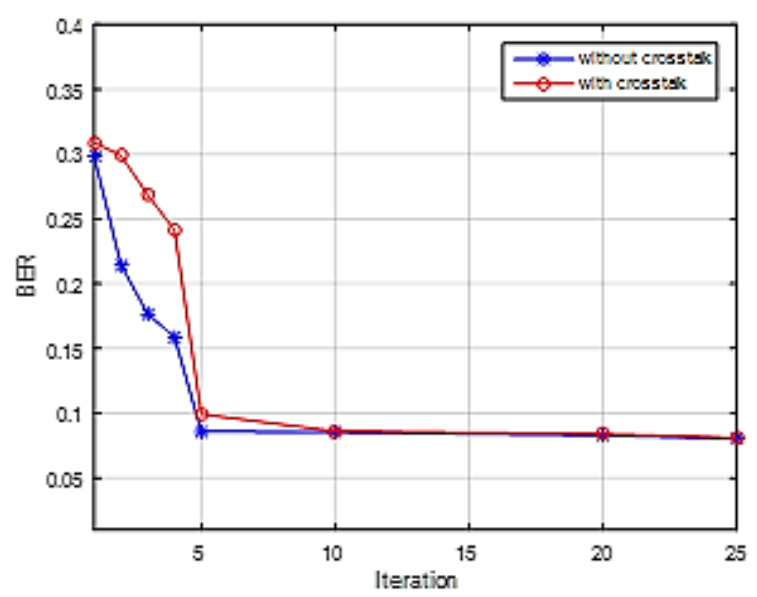

Figure. 10 BER vs iteration at IBO $7 \mathrm{~dB}$

it. However, an increase in the number of iterations provides a significant improvement in the estimation process.

Fig. 10 shows BER versus iteration for the performance of iterative receiver techniques on MIMO-GFDM systems with and without crosstalk during IBO $7 \mathrm{~dB}$. As observed, the system with an HPA and crosstalk has worse performance than the one without it.

However, the iterative receiver technique can compensate for the combined effects of nonlinear and crosstalk distortion on the MIMO-GFDM system after more than 1 iteration. When compared with the pre-distorter technique in [8], the proposed method has a better ability to compensate for the combined effects and the major advantage is that it can be applied at the base station. Therefore, there is no increase in the computational complexity on the mobile terminal, costs, or battery life. It is also applied to the receiver and compensates for the combined effects in the analyzed systems. Moreover, in comparison with the technique proposed in [4], it can be applied to non-orthogonal multicarrier systems.

\section{Conclusion}

An iterative receiver method was proposed in MIMO-GFDM systems to compensate for the combined effects of nonlinear and crosstalk distortion. The iterative receiver is able to compensate for the combined effects of crosstalk and nonlinear distortion in small iterations, ie 1-4 for mapping 16 OQAM. The advanced nature of the technique presented is that there is no need to make any changes on the cellular terminal side. The method was also shown to have the ability to compensate for the combined effects which no previous research has been able to examine. Future research is needed to apply this technique to overcome the combination of crosstalk and nonlinear distortion in the MIMO-GFDM system in real experiments.

\section{Conflicts of Interest}

The authors declare no conflict of interest.

\section{Author Contributions}

Conceptualization, A.E.J. and W.; methodology, A.E.J., W., and T.S.; software, A.E.J. and T.S.; validation, A.E.J. and W.; formal analysis, A.E.J., W., and T.S..; investigation, A.E.J., W., and T.S.; resources, A.E.J; data curation, A.E.J.; writingoriginal draft preparation, A.E.J.; writing - review and editing, A.E.J., W., T.S., and E.; visualization, A.E.J. and W.; supervision, W., T.S., and E.; project administration, A.E.J., W., T.S., and E.; funding acquisition, A.E.J., W., T.S., and E.

\section{Acknowledgments}

The authors acknowledge the support for this research from the scholarship program BUDI-DN by 
the LPDP of the Ministry of Finance of the Republic of Indonesia to Ari Endang Jayati.

\section{References}

[1] A. Hammoodi, L. Audah, and M. A. Taher, "Green Coexistence for 5g Waveform Candidates: A Review", IEEE Access, Vol. 7, pp. 10103-10126, 2019.

[2] J. Demel, C. Bockhelmann, and A. Dekorsy, "Evaluation of a Software Defined GFDM Implementation for Industry 4.0 Applications", In: Proc. of 2017 IEEE International Conference on Industrial Technology (ICIT), Toronto, Canada, pp. 1283-1288. 2017.

[3] R. N. Hasyim and E.Y. Hammid. "Carrier Frequency Offset Estimation Using Cyclic Prefix in GFDM Systems for Machine Type Communication Application", In: Proc. of 2018 8th International Conference on Intelligent Systems, Modelling and Simulation Carrier, Kuala Lumpur, Malaysia, pp. 126-130, 2018.

[4] N. A. El-Alfi, H. M. Abdel-atty, and M. A. Mohamed, "Sub-Nyquist Cyclostationary Detection of GFDM for Wideband Spectrum Sensing", IEEE Access, Vol. 7, pp. 8640386411, 2019.

[5] Z. Na, Z. Pan, M. Xiong, X. Liu, W. Lu, Y. Wang, and L. Fan, "Turbo Receiver Channel Estimation for GFDM-Based Cognitive Radio Networks", IEEE Access, Vol. 6, pp. 9926-9935, 2018.

[6] Y. Yang and L. Zhu, "A Data-Aided Interference Cancellation GFDM Receiver for Hybrid Satellite-Terrestrial Communication Systems", In: Proc. of 2018 International Symposium on Networks, Computers and Communications (ISNCC), Rome, Italy, pp. 1-6, 2018.

[7] R. M. Borges, T. R. R. Marins, M. S. B, Cunha, H. R. D. Filgueiras, I. F. da Costa, R. N. da Silva, D. H. Spadoti, L. L. Mendes, and A. C. Sodre, "Integration of a GFDM-Based 5G Transceiver in a GPON Using Radio over Fiber Technology", Journal of Lightwave Technology, Vol. 36, No. 19, pp. 4468-77, 2018.

[8] Z. A. Sim, F. H. Juwono, R. Reine, Z. Zang, and L. Gopal, "Performance of GFDM Systems Using Quadratic Programming Pulse Shaping Filter Design", IEEE Access, Vol. 8, pp. 3713437146, 2020.

[9] K. Gumber and M. Rawat, "A Modified Hybrid RF Predistorter Linearizer for Ultra Wideband 5G Systems", IEEE Journal on Emerging and
Selected Topics in Circuits and Systems, vol. 7, no. 4, pp. 547-557, 2017.

[10] L. Cho, C. H. Ting, C. Y. Chen, C. H. Yau, C. Y. Hsu, "When UUDN Meets 5G Waveforms: New Challenge of Signal Linearity?", In: Proc. of the 2017 IEEE International Conference on Applied System Innovation: Applied System Innovation for Modern Technology, Taiwan, China, pp. 770-773, 2017.

[11] P. Jantunen, "Modelling of Nonlinear Power Amplifiers for Wireless Communications", M.S. thesis, Dept. Electron. And Comm. Eng., Helsinky University of Technology, Finland, 2004.

[12] F. Gregorio, S. Werner, T. I. Laakso, and J. Cousseau, "Receiver Cancellation Technique for Nonlinear Power Amplifier Distortion in SDMA - OFDM Systems", IEEE Transactions on Vehicular Technology, Vol. 56, No. 5, pp. 2499-2516, 2007.

[13] A. E. Jayati, Wirawan, T. Suryani, and Endroyono, "Characteristic of HPA Nonlinear Distortion Effects in MIMO-GFDM Systems", In: Proc. of the 9th International Conference on Information and Communication Technology Convergence: ICT Convergence Powered by Smart Intelligence, Jeju Island, Korea, pp. 379384, 2018.

[14] G. U. Maheswari, A. Govindasamy, and S. J. Thiruvengadam, "Performance Analysis of Filter Bank Multicarrier System with NonLinear High Power Amplifiers for 5G Wireless Networks", IET Signal Processing, Vol. 11, No. 1, pp. 66-72, 2017.

[15] M. C. Dakhli, R. Zayani, O. B. Belkacem, and R. Bouallegue, "Theoretical Analysis and Compensation for the Joint Effects of HPA Nonlinearity and RF Crosstalk in VBLAST MIMO-OFDM Systems over Rayleigh Fading Channel", Eurasip Journal on Wireless Communications and Networking, Vol. 1, No. 61, pp. 1-15, 2014.

[16] A. Ortega, L. Fabbri, and V. Tralli, "Performance Evaluation of GFDM over Nonlinear Channel", In: Proc. of 2016 International Conference on Information and Communication Technology Convergence, Jeju Island, Korea, pp. 12-17, 2016.

[17] A. E. Jayati, Wirawan, and T. Suryani, “Analysis of Non-Linear Distortion Effect Based on Saleh Model in GFDM System", In: Proc. of 2017 IEEE International Conference on Communication, Networks and Satellite, Semarang, Indonesia, pp. 1-6, 2017. 
[18] M. Matthé, L. Mendes, I. Gaspar, N. Michailow, D. Zhang, and G. Fettweis, "Precoded GFDM Transceiver with Low Complexity Time Domain Processing", Eurasip Journal on Wireless Communications and Networking, Vol. 138, pp. 1-9, 2016.

[19] A. Hilario-tacuri, J. M. P. Fortes, R. SampaioNeto, L. Soncco, D. Donaires, and J. Borja, "Performance Evaluation of Generalized Frequency Division Multiplexing Systems Over Non-Linearities with Memory", IEEE Access, Vol. 7, pp. 119131-119139, 2019.

[20] D. Falconer, F. Danilo-Lemoine, C. -T. Lam, and M. Sabbhagian, "Power Backoff Reduction for Generalized Multicarrier Waveforms", In: Proc. of European Signal Processing Conference, Poznan, Poland, Vol. 2008, pp. 693-697, 2007.

[21] S. S. K. C. Bulusu, H. Shaiek, and D, Roviras, "PA Linearization of FBMC-OQAM Signals with Overlapped Recursive Error Correcting Predistortion", In: Proc. of the International Symposium on Wireless Communication Systems (ISWCS) 2016, Lisbon, Portugal, pp. 533-537, 2016.

[22] K. K. Kumar, "Improved PTS Technique Based on Sub-Block Weighting Method of PAPR Reduction in OFDM Signals", International Journal of Intelligent Engineering and Systems, Vol. 10, No. 5, pp. 87-94, 2017.

[23] A. E. Jayati, Wirawan, T. Suryani, and Endroyono, "Partial Transmit Sequence and Selected Mapping Schemes for PAPR Reduction in GFDM Systems", International Journal of Intelligent Engineering and Systems, Vol. 12, No. 6, pp. 114 - 122, 2019.

[24] L. Sendrei and S. Marchevsky, "Nonlinear Noise Estimation and Compensation in GFDM Based Communication Systems for Cognitive Radio Networks", In: Proc. of Radioelektronika (RADIOELEKTRONIKA), $2015 \quad$ 25th International Conference, Pardubice, Czech Republic, pp. 313-316, 2015.

[25] A. Vaezi, A. Abdipour, A. Mohammadi, and F. Ghannouchi, "Analysis of Nonlinear Crosstalk Impairment in MIMO-OFDM Systems", Analog Integrated Circuits and Signal Processing, Vol. 99, No. 3, pp. 559-569, 2019.

[26] D. Korpi, Dani, L. Antilla, and M. Valkama, "Nonlinear Self-Interference Cancellation in MIMO Full-Duplex Transceivers under Crosstalk", EURASIP Journal on Wireless Communications and Networking, Vo. 24, pp. 115, 2017.
[27] F. H. Gregorio, S. Werner, J. Cousseau, J. Figueroa, and R. Wichman, "Power Amplifier Linearization Technique with IQ Imbalance and Crosstalk Compensation for Broadband MIMOOFDM Transmitters", EURASIP Journal on Advances in Signal Processing, Vol. 91, No. 8, pp. 2042-2056, 2011.

[28] S. A. Bassam, M. Healoui, and F. M. Ghannouchi, "Crossover Digital Predistorter for the Compensation of Crosstalk and Nonlinearity in MIMO Transmitters", IEEE Transactions on Microwave Theory and Techniques, Vol. 57, No. 5, pp. 1119-1128, 2009.

[29] A. E. Jayati, Wirawan, T. Suryani, and Endroyono, "Nonlinear Distortion Cancellation Using Predistorter in MIMO-GFDM Systems", Electronics, Vol. 8, No. 620, pp. 1-19, 2019.

[30] A. A. M, Saleh, "Frequency-Independent and Frequency-Dependent Nonlinear Models of TWT Amplifiers", IEEE Transactions on Communications, Vol. 29, No. 11, pp. 17151720, 1981.

[31] P. Wei, X. Xia, Y. Xiao, and S. Li, "Fast DGTBased Receivers for GFDM in Broadband Channels", IEEE Transactions on Communications, Vol. 64, No. 10, pp. 43314345, 2016.

[32] S. K. Bandari, V. V. Mani, and A, Drosopoulos, "GFDM / OQAM Implementation under Rician Fading Channel", In: Proc. of the Intl. Conference on Advances in Computing, Communications and Informatics (ICACCI), Jaipur, India, pp. 256-260, 2016.

[33] J. Qi, "Analysis and Compensation of Channel and RF Impairments in MIMO Wireless Communication Systems", Ph.D, Dissertation, Institut National De La Recherche Scientifiqu, Canada, 2011. 